awareness and/or availability of targeted support services to aid patients with their recovery.

Doctors are knowledgeable to varying degrees about the impact of the recovery process on patients. When managing patient's expectations of recovery, it is evident that doctors provide general advice based on their clinical experience, rather than evidence. Many also noted that patients with CAP seek reassurance, often afraid of recurrence upon hospital discharge.

Conclusion Doctors have mixed views on the current management of patients with CAP following discharge. Many concurred that patients experience anxiety and morbidity associated with recovery from CAP. Also, a perceived deficiency in the support infrastructure for affected patients was noted. Thus, further research could consider post-discharge management and recovery, in order to improve existing clinical recommendations and resources.

\section{P34 PARAMEDIC TRAIL BLAZERS - WHY DO PARAMEDICS TAKE PART IN PREHOSPITAL RESEARCH?}

${ }^{1}$ BA Evans*, ${ }^{2} \mathrm{~A}$ Brown, ${ }^{1} J$ Bulger, ${ }^{1} \mathrm{G}$ Fegan, ${ }^{3} \mathrm{~S}$ Ford, ${ }^{3} \mathrm{~K}$ Guy, ${ }^{2} \mathrm{~S}$ Jones, ${ }^{4} \mathrm{~L}$ Keen, ${ }^{4} \mathrm{~N}$ Rees, ${ }^{1} \mathrm{H}$ Snooks. ${ }^{1}$ Medical School, Swansea University, Swansea, UK; ${ }^{2}$ Public contributor, clo Swansea University, UK; ${ }^{3}$ Abertawe Bro Morgannwg University Health Board, Swansea, UK; ${ }^{4}$ Welsh Ambulance Service NHS Trust, Swansea, UK

\subsection{6/jech-2018-SSMabstracts. 160}

Background Research to improve prehospital treatment and care requires the participation of clinical and managerial staff from ambulance services to test interventions and collect research data. Researchers work closely with individuals and organisations with prehospital clinical, managerial and policy expertise to plan, deliver and disseminate research evidence. A recent trial involved assessing feasibility of paramedics administering Fascia Iliaca Compartment Block (FICB) in place of morphine to patients with suspected hip fracture. Participation in the trial was voluntary. We explored paramedics' motivation for participating in research through the RAPID (Rapid Analgesia for Prehospital Hip Disruption) trial.

Methods We held three focus groups with 11 RAPID trial paramedics serving one district hospital, audio-recorded with participants' consent. We conducted thematic analysis of interview transcripts. Two researchers, one paramedic and one lay member were in the analysis team.

Results Paramedics believed their participation in research had benefits for patients, for them individually and for the paramedic profession. Respondents said that being part of a research project provided an opportunity to increase their skills and provide improved patient care. In the RAPID trial, they understood the potential risks and benefits of different medications and management for patients with suspected hip fracture and welcomed the chance to identify improved pain management for a vulnerable population. They felt proud to be learning and using a procedure usually administered by clinical staff: 'This is quite specialised, like, hang on, I'm doing a really top job here, dealing with anaesthetics...blunt needles... it's like 'up there'. It's not our everyday thing.'

More generally, respondents identified how research potentially contributed towards widening the scope of paramedic practice by extending their role into clinically specialist areas. They suggested that many paramedics were unwilling to undertake tasks perceived to exceed their pay-grades and shunned the opportunity to learn new techniques which were not routine care. In comparison, these paramedics saw themselves as trail-blazers for the profession, proud to be acquiring extra clinical responsibilities and skills. They also valued the chance to contribute to the evidence base, providing knowledge about using alternative medication which they instinctively felt would cause fewer complications and benefit patient mortality and morbidity.

Conclusion Paramedics who take part in research believe they contribute to personal and professional development and potentially improve patient care. Future research with paramedics refusing research participation could explore barriers and different views on skills development, which may also inform implementation of new evidence-based interventions.

\section{\begin{tabular}{|l|l}
\hline P35 IS THE STORY ABOUT WORRYING WOMEN AND \\
\hline
\end{tabular} STOICAL MEN TRUE? GENDER DIFFERENCES IN SUBJECTIVE HEALTH AFTER ADJUSTMENT FOR REPORTING STYLES}

\begin{abstract}
${ }^{1}$ A Oksuzyan*, 'MJ Dańko, ${ }^{2,3}$ D Jasilionis, ${ }^{2,4}$ V Shkolnikov. 'Max Planck Research Group, Max Planck Institute for Demographic Research, Rostock, Germany; 'Laboratory of Demographic Data, Max Planck Institute for Demographic Research, Rostock, Germany; ${ }^{3}$ Demographic Research Centre, Vytautas Magnus University, Kaunas, Lithuania; ${ }^{4}$ International Laboratory for Population and Health, National Research University Higher School of Economics, Moscow, Russian Federation
\end{abstract}

\subsection{6/jech-2018-SSMabstracts. 161}

Background Although women are less healthy than men with respect to physical health and depression, gender differences in morbidity and self-rated health $(\mathrm{SRH})$ are less consistent. Female disadvantage in SRH has been partially explained by women's preponderance to report poor health. Although more recent studies challenge gender stereotypical treatment-seeking behavior and reporting of specific health conditions, we know relatively little about gender differences in reporting of SRH. The present study investigates to which extent adjusting for differences in reporting styles modifies gender differences in $\mathrm{SRH}$, whether these changes are due to gender-specific overand/or under-reporting patterns, and whether these changes are consistent for reporting of poor and good health.

Methods We utilize the data collected in the wave 1 (2004) of the Survey of Health, Ageing and Retirement in Europe, a multidisciplinary panel survey of community-dwelling individuals aged $50+$ in 12 countries. The analysis follows the method proposed by Jürges (2007) and Rebelo and Pereira (2014). Our dependent variable is a global evaluation of health 5 possible responses: excellent, very good, good, fair, and poor. SRH is our dependent variable and is modelled with a generalized ordered probit model. There are two types of independent variables: latent health (e.g. chronic conditions, physical limitations, and smoking) variables and threshold variables (gender, education level and country). Once the appropriate model is selected and fitted, health indexes and disability weights are calculated. They, in turn, are used to calculate SRH adjusted for reporting styles.

Results Our preliminary analyses suggest that when SRH is adjusted for differences in reporting styles, the gender gaps in the percentage of persons with both poor and good widen. Except the oldest age group, more men have reported poor health than they have, whereas less women have reported poor SRH than they have except the youngest women. These findings suggest that men over-report and women under-report poor health. At all ages less men have reported very good 